\title{
Joel F. Harrington, Die Ehre des Scharfrichters. Meister Frantz oder ein Henkersleben im 16. Jahrhundert
}

\section{Falk Bretschneider}

\section{OpenEdition}

\section{Journals}

Édition électronique

URL : http://journals.openedition.org/ifha/8725

DOI : $10.4000 /$ ifha. 8725

ISSN : 2198-8943

Éditeur

IFRA - Institut franco-allemand (sciences historiques et sociales)

Référence électronique

Falk Bretschneider, « Joel F. Harrington, Die Ehre des Scharfrichters. Meister Frantz oder ein Henkersleben im 16. Jahrhundert », Revue de l'IFHA [En ligne], Date de recension, mis en ligne le 02 novembre 2017 consulté le 24 septembre 2020. URL : http://journals.openedition.org/ifha/8725 ; DOI : https://doi.org/ $10.4000 /$ ifha. 8725

Ce document a été généré automatiquement le 24 septembre 2020.

(C)IFHA 


\section{Joel F. Harrington, Die Ehre des Scharfrichters. Meister Frantz oder ein Henkersleben im 16. Jahrhundert}

Falk Bretschneider

\section{RÉFÉRENCE}

Joel F. Harrington, Die Ehre des Scharfrichters. Meister Frantz oder ein Henkersleben im 16. Jahrhundert, München: Siedler, 2014, 399 p., 24,99€ 
Meister Frantz - maître Frantz, de son nom complet Frantz Schmidt (1555-1634) - de Nuremberg fut certainement le bourreau le plus connu de l'Allemagne moderne, comparable par sa dimension quasi mythique à Charles-Henri Sanson (de la fameuse dynastie des exécuteurs parisiens), qui partage avec lui la particularité d'avoir laissé à la postérité un témoignage de soi qui n'a cessé de fasciner les historiens. Contrairement à Sanson, dont les mémoires sont considérés comme apocryphes, le journal intime de Schmidt, tenu entre 1573 (année de sa première pendaison) et 1618 (moment de son retrait de la vie active), est véritablement de sa main et recense, en des notes souvent sommaires, l'ensemble des 394 exécutions capitales et des centaines de mutilations ou bannissements qu'il a accomplis au cours

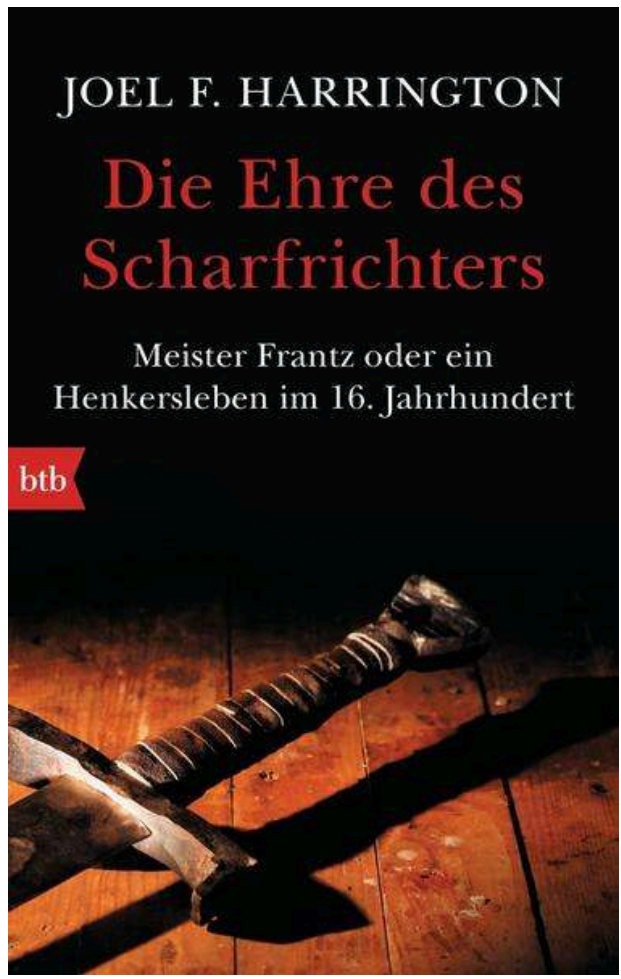
de sa carrière (p. 13). Ce journal est certes connu des historiens : bien que l'original soit perdu, plusieurs copies existent dans les archives, et il fut imprimé en 1801 et en 1913 (et réédité plusieurs fois par la suite). Mais jusqu'à présent, personne n'avait essayé d'extraire de cette énumération assez macabre de malfaiteurs décapités, pendus, roués ou brûlés vifs, la vision du monde de maître Frantz lui-même.

Deux circonstances ont incité Joel Harrington, professeur à l'Université Vanderbilt à Nashville et connu pour ses travaux sur l'Allemagne moderne, à tenter ce que d'autres avant lui n'avaient pas osé faire: d'une part la découverte d'une nouvelle copie du journal, datant de 1634 (l'année de la mort du bourreau) et exempte d'un nombre considérable d'ajouts ou d'altérations greffés sur les copies ultérieures; d'autre part une supplique, conservée aujourd'hui au Haus-, Hof- und Staatsarchiv de Vienne, et que l'ancien exécuteur, presque septuagénaire, adressa en 1624 à l'empereur Ferdinand II. Dans ce dernier document, maître Frantz réclamait le rétablissement de son honneur et de celui de sa famille, mis à mal en 1553 lorsque son père Heinrich, estimable forestier princier, avait été contraint par Albert Alcibiade, redoutable margrave de Brandebourg-Kulmbach, à exécuter trois prétendus comploteurs - ce qui avait eu pour effet immédiat de le condamner, ainsi que sa descendance, à l'infamie d'une triste tradition professionnelle: celle du bourreau. La volonté d'annuler ce malheureux événement et le souhait de rendre à sa famille une place au sein de la société honorable représentent selon Harrington la clé nécessaire pour comprendre l'existence de maître Frantz qui mit toute sa vie, et son journal, au service de cet objectif.

À travers cinq chapitres, le livre retrace donc la biographie fascinante d'un homme qui, en accomplissant un métier qu'il détestait par-dessus tout avec le plus grand sérieux et une discipline remarquable (ce qui fut loin d'être le cas de tous ses collègues), posa les bases lui permettant d'obtenir, par une vie irréprochable au niveau privé comme 
professionnel, sa réintégration dans la bonne société. Nous découvrons certains traits de la jeunesse du futur bourreau qui grandit à Bamberg (et s'entraînait, sous l'œil critique de son père, à la décapitation sur des courges ou des chiens), l'accompagnons lors de ses pérégrinations dans la région (destinées à le perfectionner dans la « mise en scène d'une belle mort ", p. 124), et sommes à ses côtés lorsque Schmidt fut nommé, en janvier 1577 et grâce aux efforts de son père, bourreau en titre de la ville impériale de Nuremberg, l'un des postes les plus convoités de tout l'Empire. Comptant parmi les «métropoles» du Reich, la ville franconienne offrait à son bourreau de nombreuses occasions de montrer son savoir-faire (contrairement à beaucoup de ses confrères, souvent enclins à l'alcoolisme pour s'accommoder de leur position sociale, Schmidt ne rata que très peu des exécutions qui lui furent confiées), compétence que les pères de la ville rétribuèrent en lui accordant un salaire qui en fit l'exécuteur le mieux payé d'Allemagne (et le propulsa parmi les cinq pour cent de salariés les mieux rémunérés de la ville, p. 154).

C'est cette position qui permit à Schmidt de fonder une famille et d'établir les bases de son retour dans la société honorable, notamment par un travail et un comportement personnel qui, en élevant l'honnêteté au rang de la première des vertus, lui permit de construire son parcours autour d'une qualité dont il avait la maîtrise (p.186). En affichant une fidélité infaillible envers les autorités, en partageant les principes moraux de la société dans laquelle il vivait et en se montrant utile à ses voisins (par une activité de chirurgien et de guérisseur à laquelle J. Harrington consacre un chapitre entier), Schmidt réussit à acquérir pas à pas la place qu'il considérait comme étant la sienne. Le premier succès de ses efforts fut l'obtention, en juillet 1593, des pleins droits d'un citoyen nurembergeois; l'ultime fut le soutien que le Conseil accorda à sa supplique visant au rétablissement de son honneur et de celui de sa famille, en faisant valoir « sa vie retirée et tranquille et sa médecine efficace, de même sa connaissance du droit romain impérial» (p. 317). Le 10 septembre 1624, l'ancien bourreau put faire enregistrer le privilège impérial à la chancellerie nurembergeoise et offrir ainsi à ses fils un avenir honorable de chirurgiens (et à sa fille le mariage avec un graveur reconnu dans la ville). Schmidt succomba à la peste dix ans plus tard, en 1634, et fut enterré dans le cimetière municipal (où sa tombe existe toujours), apparaissant dans les papiers officiels comme « l'honorable Frantz Schmidt, médecin ».

Avec ce livre, J. Harrington ne réussit pas seulement à reconstituer cet incroyable parcours d'un homme décidé, il démontre également - à l'instar des travaux de Jutta Nowosadtko - qu'il n'est pas satisfaisant de se contenter d'inscrire le bourreau parmi les nombreux marginaux de la société moderne. Le « va-et-vient de personnes de toutes qualités » venant chercher des conseils médicaux et la guérison dans la maison de l'exécuteur (p. 282) montre que la position sociale des membres de cette profession était plus complexe; évités au grand jour et appréciés dans l'intimité pour leurs connaissances et leurs capacités thérapeutiques, ils vivaient dans un état flottant entre le rejet et l'acceptation dissimulée. Mais surtout, et l'exemple de maître Frantz le montre parfaitement, leur statut n'était pas gravé dans le marbre; le bourreau de Nuremberg réussit à s'extraire de sa position infamante, démontrant ainsi qu'une mobilité sociale était possible, fût-ce au prix d'efforts considérables. Initialement paru en 2013 à New York, le livre se présente ici dans l'excellente traduction allemande de Norbert Juraschitz qui reproduit à merveille le style agréable de J. Harrington. Celui-ci sait construire un récit qui captive son lecteur en n'hésitant pas à combler les lacunes des sources par telle ou telle scène imaginée (en s'appuyant évidemment sur l'état des 
connaissances). Certains critiques allemands ont vu dans cette liberté littéraire une tendance aguicheuse (par exemple Valentin Groebner dans la $F A Z$ du 9 juillet 2014); nous y voyons le talent d'un excellent historien mêlé à celui d'un excellent narrateur, de sorte que l'on n'apprend pas seulement beaucoup de choses dans ce livre, mais qu'on le fait en prenant du plaisir. Qu'attendre davantage d'un bon livre d'histoire?

INDEX

Thèmes : Histoire sociale, Histoire des mentalités, Histoire du droit Index chronologique : Période moderne

\section{AUTEURS}

FALK BRETSCHNEIDER

EHESS Paris 